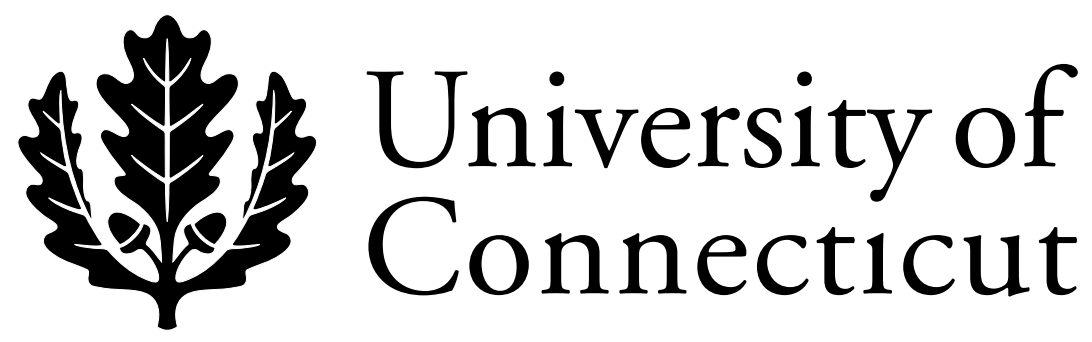

Department of Economics Working Paper Series

\title{
Legislative Bargaining and Incremental Budgeting
}

Dhammika Dharmapala

University of Connecticut

Working Paper 2002-10

October 2002

341 Mansfield Road, Unit 1063

Storrs, CT 06269-1063

Phone: (860) 486-3022

Fax: (860) 486-4463

http://www.econ.uconn.edu/ 


\begin{abstract}
The notion of incrementalism, formulated by Aaron Wildavsky in the 1960s, has been extremely influential in the public budgeting literature. In essence, it entails the claim that legislators engaged in budgetary policymaking accept past allocations, and decide only on the allocation of increments to revenue. Wildavsky explained incrementalism with reference to the cognitive limitations of lawmakers and their desire to reduce conflict. This paper uses a legislative bargaining framework to undertake a formal analysis of incremental budgeting. An exogenously chosen agenda setter proposes budgets and seeks to build coalitions to secure passage, over multiple periods. The central result is that the agenda setter can lower her cost of building a winning coalition, and thereby raise her payoff, by following an incrementalist strategy, which involves maintaining the same coalition every period. First, it is shown within a simple 2-period model that there exist subgame perfect incremental budgeting equilibria of this nature. If the agenda setter is assumed to be able to commit to the grandfathering of past allocations, the unique subgame perfect equilibrium (up to the choice of the coalition members) involves incremental budgeting. The model is then extended to an infinitely repeated setting, and it is shown that the agenda setters incentives for incremental budgeting are reinforced in this context. Some testable implications (relating incrementalism to various characteristics of the legislature) are also derived. Finally, the implications for incrementalism of heterogeneity among legislators are analyzed.
\end{abstract}

Journal of Economic Literature Classification: D72, H61

Keywords: Legislative bargaining; incremental budgeting, budgetary institutions 


\section{1) Introduction}

Ever since it was proposed by Aaron Wildavsky (e.g. 1984; 1988) in the 1960's, 'incrementalism' has proven to be an extremely influential theory within the field of public budgeting. ${ }^{1}$ Incrementalism is defined as "a process in which budgetary bases (i.e. previous expenditures) are accepted ... [and] budgeting is a stable process ..." (Gist, 1982, p. 859). This theoretical framework encompasses a descriptive claim as well as a normative one. The former involves the contention that Congress and other legislatures follow an incrementalist mode of budgetary decisionmaking (or, at least, that they did so during the period in which Wildavsky developed the theory). The latter claim rests on Wildavsky's (1984, p. 136) argument that incremental budgeting is superior to feasible alternatives because of the cognitive limitations of lawmakers, and because it can attenuate political conflict over budgeting priorities. Although the theory has declined in salience in the United States since the 1980's due to various changes in the budgetary environment, ${ }^{2}$ it remains an important account of 'classical' or 'traditional' budgeting in the public budgeting literature.

While there have been extensive conceptual discussions and empirical studies of this topic, little attempt has previously been made to examine the implications of formal gametheoretic models of legislative institutions for the analysis of incremental budgeting. The aim of this paper is to develop such an analysis, using a modified version of the legislative bargaining framework of Baron and Ferejohn (1989). ${ }^{3}$ The model assumes a legislature with an exogonously given agenda setter, who has the power to propose budget allocations over multiple periods. In order to secure passage of the budget each period, the agenda setter must attract the support of a given number of the other ('junior') legislators. She obtains their votes by allocating funds for their districts in the budget proposal. The requirement that the budget must be passed, and the available revenue, constrain the agenda setter in seeking to maximize the budgetary allocation for her own district.

\footnotetext{
${ }^{1}$ According to Berry (1990, p. 167): "No single concept has been more central to the study of public budgeting over the last three decades."

${ }^{2}$ Among the most important of these changes was that the budgetary increments of the past were transformed into decrements - both due to the deficits of the 1980's, and to the growth of entitlement spending, which reduced the revenue available for discretionary allocation. Thus, Garrett (1998, p. 392, note 14) notes that incrementalism was "easier to maintain when federal budgets seemed to be continually increasing", while "[t]he salience of the budget deficit and the disappearance of the increment ... undermined the theory."

${ }^{3}$ Recent examples of the use of such a model to analyze issues in public finance include Leblanc, Snyder and Tripathi (1999) and Dharmapala (1999). See also Knight (2002) for an empirical perspective.
} 
This paper uses a specific conceptualization of the notion of incrementalism. An incremental budgeting process is defined as one characterized by 'coalitional stability' (the maintenance by the agenda setter of the same coalition every period), where the budget allocations of members of this stable coalition are nondecreasing. ${ }^{4}$ The basic intuition for the results of this paper is that, by instituting incremental budgeting practices, the agenda setter can lower the cost of building a winning coalition to pass the budget, and thereby raise her payoff. In particular, under incremental budgeting, the junior legislators who are included in the stable coalition are willing to accept (and vote for) lower budget allocations each period than under alternative nonincremental procedures, in exchange for the certainty of being included in future coalitions. Thus, incremental budgeting lowers the cost (in terms of allocations to other legislators' districts) that the agenda setter incurs to secure passage of the budget, and leaves more of the budget available for allocation to her own district.

Section 3 develops a simple 2-period budgeting game of the kind described above. It is shown that there exist subgame perfect 'incremental budgeting' equilibria (in which the agenda setter maintains the same coalition in period 2 as in period 1, and the members of this coalition vote for period 1 allocations that are lower than their default payoffs). However, there also exist other ('nonincremental') equilibria, where the agenda setter's payoff is lower (and the payoffs of the other legislators are higher) than in the incremental equilibria. Then, it is assumed that the agenda setter can credibly precommit to 'grandfathering' past budget allocations, for example, through the choice of institutional features of budgetary decisionmaking, such as budget baselines or procedural rules that preclude (or impose high transactions costs on) revisiting past allocations. ${ }^{5}$ In such circumstances, the only subgame perfect equilibrium (unique up to the choice of the coalition members) involves incremental budgeting.

In Section 4, it is shown that the agenda setter's incentives for incremental budgeting are reinforced in an infinite horizon context. Moreover, numerical simulations for reasonable values of the model's parameters suggest that the magnitude of the increase in the agenda setter's perperiod payoff is substantial. Section 5 introduces heterogeneity among the junior legislators in the default payoffs they receive if the agenda setter's proposal is defeated. The result is that, if

\footnotetext{
${ }^{4}$ Clearly, this formulation fails to capture all of the myriad ways that the term 'incrementalism' has been used in the public budgeting literature; however, it is sufficiently precise to serve as a starting point for formal analysis.

${ }^{5}$ For an empirical analysis of the consequences of budget baselines for fiscal policy, see Crain and Crain (1998).
} 
the differences in the default payoffs are sufficiently small, then, an agenda setter who can credibly precommit to grandfathering can increase her payoff by using incremental budgeting.

These results imply that budget proposers may have an incentive to institute incremental budgeting practices in order to lower their costs of passing the budget, thereby increasing the revenue available for their own preferred spending priorities. This explanation for incrementalism contrasts with Wildavsky's emphasis on cognitive limitations and the desire to reduce conflict. The model also yields some testable predictions linking this incentive for incremental budgeting to factors such as the size of the legislature, supermajority requirements, and the discount rate.

Following this introduction, Section 2 elaborates further on the idea of incrementalism. The basic 2-period model is presented in Section 3, and Section 4 develops the infinitely repeated version of the model. The extension to heterogeneous legislators is presented in Section 5. Section 6 concludes the paper, and discusses some further possible extensions.

\section{2) The Notion of Incremental Budgeting}

The notion of incremental budgeting is closely associated with Aaron Wildavsky's characterization of 'classical budgeting'. While there are many different definitions of incrementalism (see e.g. Berry (1990)), the basic feature is that budgetary allocations are relatively stable over time. The allocation received by a particular area for one year forms the basis of its allocation for the next, and decisionmaking focuses on increments to revenue. Thus, Wildavsky (1984, p. xii) characterizes incrementalism as a process "where changes are small, alternatives resemble those of the past, and patterns of relationships among participants remain stable."

Wildavsky's formulation, as developed in his classic 1964 book, The Politics of the Budgetary Process, and in his subsequent writings, has both positive and normative components. The positive, or descriptive, aspect of his theory is summarized as follows: "The largest determining factor of this year's budget is last year's. Most of each budget is a product of previous decisions ... many items are standard, simply reenacted every year” (1988, p. 78). Moreover, "[a]t any one time, after past commitments are paid for, a rather small percentage - 
seldom larger than 30 percent, often smaller than 5 - is within the realm of anybody's . . . discretion as a practical matter" $(1988$, p. 78$){ }^{6}$

The major alternatives to budgetary incrementalism that have been proposed by scholars of public budgeting are 'zero-based budgeting' (ZBB) and 'planning, programming, and budgeting systems' (PPBS), also known as 'program budgeting'. The former involves reconsidering budgetary allocations in their entirety each year, using a zero base for each program. The latter involves focusing on the goals and objectives of budgetary expenditures (i.e. the outputs of government programs) and comparing different expenditure packages in terms of their success in producing these outputs.

The normative aspect of Wildavsky's theory entails the claim that incremental budgeting is superior to these 'comprehensive' approaches; two main reasons are offered. One is that incrementalism "increases agreement among the participants" (1984, p. 136), and can thus promote consensus (or at least suppress conflict) among budgetary policymakers. The other is that incrementalism reduces the "burden of calculation" (1984, p. 136) and imposes fewer demands on legislators' cognitive capacities. ${ }^{7}$ In this vein, he argues that: "Clinging to last year's agreements is enormously economical of critical resources . . . which would be seriously depleted if all or most past agreements were reexamined yearly." (1984, p. 217).

Thus, Wildavsky's explanation for why legislators may favor an incremental approach to budgeting relies primarily on their information-processing limitations, as well as on their desire to avoid conflict. These motivational assumptions are quite distinct from those that are highlighted in the rational choice literature (notably, the desire to maximize the probability of reelection and to obtain redistributive transfers). Indeed, the issue of incremental budgeting has attracted little analysis from a rational choice perspective. In particular, while there has been some recent literature on the impact of interest group lobbying on incremental budgetary outcomes (Tohamy, Aranson and Dezhbaksh, 1999; 2000), ${ }^{8}$ no previous attempt has been made

\footnotetext{
${ }^{6}$ Wildavsky (1984, p. 14) examines appropriations for a sample of 37 agencies, and shows that the variation for the majority was less than $10 \%$ per year. See also Davis, Dempster, and Wildavsky (1966) and Kamlet and Mowery (1987) for further evidence. However, Gist (1982) finds a contrary result.

${ }^{7}$ This idea could perhaps also be formulated in terms of the transaction costs of reaching a budgetary agreement.

${ }^{8}$ Tohamy et al. (1999; 2000) develop an interest group approach, in which a single legislator (who is assumed to have unilateral control of budgetary policy) is lobbied by interest groups seeking budget allocations. The legislator can choose between 'single-period budgeting', allocating funds for one period only, or 'multi-period budgeting', allocating funding in perpetuity; the latter tends to lead to incremental outcomes. Interest groups are generally willing to pay more (in campaign contributions) for a stream of future benefits, so the legislator trades off these higher contributions against the loss of flexibility entailed by incrementalism. The legislator's optimal policy
} 
to analyze incrementalism from the perspective of formal game-theoretic models of legislative institutions.

\section{3) The Basic Model}

\section{1) Assumptions}

This section presents a simple model that illustrates the main themes of the paper. The model assumes a legislature with an odd number $n$ of members. The legislature is denoted as the set $L$ (so that card $(L)=n$ ). It is assumed that the legislature operates under a rule requiring $(q+1)$ votes (out of $n)$ for the passage of legislation, where $q<(n-1)$ (i.e. the legislature does not have a requirement of unanimity). The task faced by the legislature is to enact a budget (allocating funds to each of $n$ areas) for each of two periods. The revenue available in each period is assumed to be exogenously fixed, and denoted by $R$ in period 1 , and $(R+I)$ in period 2 , where $I \geq 0$ is the incremental revenue gained in period 2. ${ }^{9}$ The (common) discount factor is given by $\delta \in(0,1]$. Time periods are indexed by $i$, and the legislators by $j$. The budget enacted in period $i$ is denoted $\mathbf{b}^{L i}$. Legislator $j$ 's payoff in period $i$ is simply the allocation $b_{j}^{L i}$, the amount that the enacted budget allocates to the $j$ 'th budgetary function. Thus, legislator $j$ 's aggregate payoff is $\left(b_{j}^{L 1}+\delta b_{j}^{L 2}\right)$. Note that the formulation here entails that the legislators are risk-neutral; generally speaking, the results below would only be reinforced if legislators were risk-averse. A natural interpretation of the model is as one of distributive politics, with the $j$ 'th element of the budget vector $\mathbf{b}^{L i}$ representing the level of spending in legislator $j$ 's district (electoral considerations are not explicitly modeled here; however, it is implicit in this interpretation that securing spending for one's district increases the probability of reelection).

In the standard legislative bargaining framework of Baron and Ferejohn (1989), nature randomly chooses a proposer from among the legislators. However, the analysis in this paper focuses on the behavior of a given agenda setter over multiple periods. Thus, it is assumed that an exogenously chosen legislator, denoted $A$, is the agenda setter for each of the two periods. Without loss of generality, $A$ is numbered legislator $j=1$; the other legislators $(j=2, \ldots, n)$ will be described as 'junior' legislators, and are collectively denoted as the set $J$ (where $\{A\} \cup J=L$ ).

depends on a variety of economic conditions. The authors also develop a new procedure for empirically testing for incremental outcomes.

${ }^{9}$ The restriction that the increments to revenue are nonnegative is not required in the infinitely repeated game of Section 4, as long as a convergence requirement is satisfied. 
Exclusive proposal power is vested in $A$, and her proposals are voted on under a closed rule (with no amendments being permitted). ${ }^{10}$ Thus, within each period, $A$ proposes a budget allocation ( $A$ 's proposals in the two periods are denoted by $\left.\mathbf{b}^{P i}(i=1,2)\right)$, and then the legislature votes on it. If it is passed, the proposal is implemented; if it is not passed, then an exogenous default budget allocation is implemented. The default allocation (or reversion point) in period $i$ is denoted by $\mathbf{b}^{D i}(i=1,2)$. Initially, it will be assumed that the $(n-1)$ junior legislators are ex ante identical, ${ }^{11}$ so that each of them receives $b_{0}{ }^{D i}$ if $A$ 's proposal is defeated in period $i$, while $A$ receives $b_{A}{ }^{D i}$; thus, $\mathbf{b}^{D i}=\left(b_{A}{ }^{D i}, b_{0}{ }^{D i} \ldots, b_{0}{ }^{D i}\right)$. Note that the (exogonous) revenue in each period must be sufficient to at least cover the default budget - thus, it is assumed that: ${ }^{12}$

and

$$
\begin{array}{r}
R \geq(n-1) b_{0}{ }^{D 1}+b_{A}{ }^{D 1} \\
R+I \geq(n-1) b_{0}{ }^{D 2}+b_{A}{ }^{D 2}
\end{array}
$$

It is assumed that $A$ 's proposals must satisfy a period-by-period budget constraint; however, permitting an intertemporal budget constraint would not substantially affect the results. ${ }^{13}$

The most straightforward interpretation of the default payoffs is as the consumption levels that are implemented when agreement is not reached within the legislature. This is similar to the model of Leblanc et al. (1999), where there are multiple periods of legislative bargaining, and a known, finite number of rounds of proposals within each period. If no proposal has passed by the end of the last round, the default is implemented for that period. It is possible to interpret the model of this paper in the same spirit as that of Leblanc et al. (1999), with the number of rounds within each period being set to 1 . This interpretation is illustrated by Figure 1.

\section{[Fig. 1 about here]}

Alternatively, it is possible to motivate the default payoffs as follows (in a manner closer to the original Baron-Ferejohn (1989) formulation). Suppose that, within each period, $A$ makes

\footnotetext{
${ }^{10}$ These institutional features are intended to reflect the Congressional budgeting context upon which Wildavsky based his theories, with $A$ playing a role analogous to the Appropriations Committee (the assumption of a closed rule need not necessarily entail a formal rule to this effect, but may reflect $A$ 's agenda setting and procedural advantages, even when an open rule nominally applies). However, the assumptions are capable of being interpreted more broadly; for instance, $A$ could be viewed as the cabinet in a parliamentary system. For models of coalition-building in parliamentary systems, see Baron (1998) and Diermeier and Merlo (2000).

${ }^{11}$ The impact of legislator heterogeneity is analyzed in Section 5.

12 The budget constraint, of course, includes the special case where the default allocation exactly exhausts the budget. The formulation in the text also allows for the possibility that the default budget is smaller in size than the available revenue. Obviously, the results below all hold for the special case as well.

${ }^{13}$ Essentially, the equilibria derived below are unchanged, except that, as $A$ discounts future payoffs, she may borrow in period 1 and thereby increase her equilibrium payoff. This would not, however, affect the comparison between incremental and nonincremental budgeting.
} 
the first proposal. If it passes, it is implemented; otherwise, $A$ loses the floor, and a junior legislator is randomly chosen to propose a budget allocation. If this proposal passes, it is implemented; otherwise, another junior legislator is randomly chosen to make a proposal, and so on (the number of rounds within a period is thus potentially infinite). Then, $b_{0}{ }^{D i}$ is the expected continuation value of the game in period $i$ to each $j \in J$, if $A$ 's proposal is rejected. A number of points concerning this interpretation are worth noting. Firstly, since the $j$ 's are ex ante identical, and given that they face identical recognition probabilities if $A$ 's proposal is rejected, $b_{0}{ }^{D i}$ should be the same for all $j$. Secondly, suppose that $A^{\prime}$ s proposal in period $1, \mathbf{b}^{P 1}$, is voted down, so that oe of the $j$ 's is chosen as proposer. In equilibrium, this $j$ can be expected to treat $A$ asymmetrically with respect to the other $j$ 's in choosing a coalition, because $A$ will have proposal power (with probability 1 ) in round 1 of period 2 . That is, equilibrium play in the game in period 1 will involve anticipating what will happen in period 2 ; hence, $b_{A}{ }^{D 1}$ will, in general differ from $b_{0}{ }^{D 1}$. Finally, if $A$ 's proposal in period $2, \mathbf{b}^{P 2}$, fails, a randomly chosen $j$ will propose without considering the 'shadow of the future'; thus, the default payoffs may differ across periods.

While the discussion above suggests that the default payoffs may differ across periods, it turns out that imposing the restriction that

A1: $b_{0}{ }^{D 1}=b_{0}{ }^{D 2}=b_{0}{ }^{D}>0 \forall j \in J$

greatly simplifies the notation and algebra, without fundamentally affecting the results; thus, this restriction will be imposed below. A more significant assumption in this model is that the default payoffs are exogenous, in the sense of being independent of the realized play of the game. In particular, this rules out the case where $b_{j}{ }^{D 2}=b_{j}^{L 1}$ (i.e. where legislator $j$ 's period 2 default is simply her period 1 allocation). While this may seem to be a significant restriction, it is argued in Section 3.6 below that, as long as $A$ can commit to grandfathering (in a sense made more precise in Section 3.4 below), then this is not so restrictive, in the sense that 'incremental' outcomes can still occur with endogenous period 2 defaults. ${ }^{14}$

Before proceeding to solve the model, it is helpful to derive two preliminary results that apply to any Nash equilibrium of the budgeting game described above. The first is closely analogous to a result in Leblanc et al. (1999, p. 35):

Lemma 1: In any Nash equilibrium, legislators resolve indifference by voting for (rather than against) a proposed budget.

\footnotetext{
${ }^{14}$ Restricting the default payoffs to be exogenous in this sense also addresses the problem that the default payoffs may not satisfy the budget constraint if they are endogenous.
} 
Proof: Suppose that legislator $j \in J$ is indifferent between voting 'yes' and 'no' for some proposed allocation that involves $b_{j}^{P I}=x$. Now suppose that $j$ resolves indifference by voting 'no'; then, $A$ can induce $j$ to vote 'yes' by setting $b_{j}^{P I}=x+\varepsilon$ (for small $\varepsilon>0$ ). However, this cannot be part of a Nash equilibrium, as $\exists$ some $\varepsilon^{\prime} \in(0, \varepsilon)$ for which $j$ will also vote 'yes', and for which $A$ will enjoy a strictly larger payoff. Thus, in any Nash equilibrium, $j$ must resolve indifference by voting 'yes'. End of Proof.

Note also that it is assumed that $A$ always votes for her own proposal. The second result is:

Lemma 2: In any Nash equilibrium, $A$ 's proposal always passes.

Proof: Consider period 1:

$R \geq(n-1) b_{0}{ }^{D}+b_{A}{ }^{D 1}$ by assumption (Equation $1 \mathrm{a}$, and $\mathrm{A} 1$ )

$\Rightarrow b_{A}{ }^{D I} \leq R-(n-1) b_{0}{ }^{D}$

To ensure passage, it is sufficient for $A$ to offer $b_{0}{ }^{D}$ to $q j$ 's; i.e. $A$ can obtain a payoff of at least $\left(R-q b_{0}^{D}\right)$ by proposing a budget that passes. As $q<(n-1)$ (by assumption), this payoff is strictly greater than $b_{A}{ }^{D 1}$. A similar argument can be made for period 2. Thus, there is no Nash equilibrium in which $A$ 's proposal fails to pass. End of Proof.

These two preliminary results are used repeatedly below.

\section{2) Solution without Commitment}

This section presents and solves a 2-period model, in which the basic game depicted in Figure 1 is played once, and then repeated once. Before proceeding, however, it is helpful to briefly consider the 1-period model, where the game in Figure 1 is played once (without repetition). $A$ 's strategy is simply a proposal $\mathbf{b}^{P 1}$, while each $j$ 's strategy maps $A$ 's proposal $b_{j}^{P l}$ into an action from the set $\{y e s$, no $\}$ (i.e. into a vote for or against the proposed budget). The relevant solution concept here (as in the later analysis) is subgame perfect Nash equilibrium. It is also generally assumed that the $j$ 's behave symmetrically when placed in identical circumstances (e.g. being offered the same allocation).

The subgame perfect equilibrium of the 1-period game involves $A$ proposing the default payoff $b_{0}{ }^{D}$ for exactly $q$ junior legislators, and 0 for all others. The $j$ 's in the coalition vote 'yes', while the others vote 'no' (this outcome follows from the following strategies being mutual best responses: $A$ proposes $b_{0}{ }^{D}$ to exactly $q$ junior legislators and 0 for all others, and each $j$ votes 'yes' whenever $b_{j}^{P 1} \geq b_{0}{ }^{D}$ and 'no' otherwise). This equilibrium is unique up to the choice of the coalition of $q j$ 's. Note that subgame perfection rules out certain Nash equilibria, such as those in which each $j$ demands strictly more than $b_{0}{ }^{D}$ to vote 'yes'. Note also that $A$ 's equilibrium payoff $\left(R-q b_{0}{ }^{D}\right)$ is greater than the default payoff $b_{A}{ }^{D 1}$. 
Now consider the 2-period game. In this section, it is assumed that $A$ cannot make binding commitments to other legislators in period 1 concerning budgetary proposals to be made in period 2. Intuitively, the prevailing budgetary institutions are assumed to entail a zero base for each area of spending. $A$ 's strategies now involve 2 proposals, $\mathbf{b}^{P 1}$ and $\mathbf{b}^{P 2}$. However, eliminating certain clearly dominated strategies (such as proposing strictly positive allocations to more than $q j$ 's, or proposing $\mathbf{0}$ ), it is readily apparent that the crucial element of $A$ 's strategy is whether or not it specifies that the same coalition will be chosen in period 2 as in period 1 . Let the period-1 and period-2 coalitions be denoted by $M_{1}$ and $M_{2}$, respectively. Then, any strategy that involves setting $M_{2}=M_{1}$ (conditional on $\mathbf{b}^{P 1}$ being enacted) will be termed an 'incrementalist' strategy (denoted $\sigma_{A}{ }^{I}$ ). Note that it will be assumed as part of such a strategy that, if $\mathbf{b}^{P 1}$ is defeated in period $1, A$ chooses $M_{2}$ randomly. This is always an optimal strategy in the remaining subgame after a first-period defeat. Essentially, it entails that $A$ cannot credibly threaten to punish those $j$ 's in $M_{1}$ who vote 'no' in period 1 by, for instance, excluding them from $M_{2}$ with probability 1. Allowing $A$ to play any (optimal) strategy following a first-period defeat, however, only reinforces the basic results of this section (see Section 3.5 for a discussion).

Now consider $j$ 's strategies. Note that subgame perfection entails that play in period 2 will always be essentially identical to the outcome of the 1-period game (discussed above): $A$ will propose $b_{0}{ }^{D}$ for exactly $q j$ 's, and these $j$ 's will vote 'yes'. Thus, $j$ 's optimal strategy in period 2 will always involve voting 'yes' to a proposal allocating $b_{j}^{P 2} \geq b_{0}{ }^{D}$, and 'no' otherwise. Taking this into account, and eliminating dominated strategies, $j$ 's strategy in the 2-period game can be characterized simply as the cut-point value of $b_{j}^{P l}$ at and above which $j$ votes 'yes' in period 1 (contingent, of course, on $A$ 's strategy in period 2). To characterize this, consider $j \in M_{I}$, and suppose $A$ is playing $\sigma_{A}{ }^{I}$ (i.e. a strategy that involves setting $M_{2}=M_{I}$ ). When $A$ 's first-period proposal $\mathbf{b}^{P 1}$ is voted on, each member of $M_{l}$ is pivotal. If she votes 'no', $\mathbf{b}^{P 1}$ is defeated; she receives the default payoff $b_{0}{ }^{D}$ in period 1 , and (in expectation) receives $(q /(n-$ 1)) $b_{0}{ }^{D}$ in period 2. If, on the other hand, she votes 'yes', $\mathbf{b}^{P I}$ is enacted; she receives $b_{j}^{P l}$ in period 1 , and $b_{0}{ }^{D}$ (with certainty) in period 2. Thus, the payoffs can be expressed as $b_{0}{ }^{D}+\delta(q /(n-1)) b_{0}{ }^{D}$ from a 'no' vote, and $b_{j}^{P I}+\delta b_{0}{ }^{D}$ from voting 'yes'. The minimum $b_{j}^{P 1}$ that will induce each $j \in$ $M_{l}$ to vote 'yes' is denoted $x^{*}$, and is obtained by equating the payoffs from voting 'yes' and 'no'; it can be characterized as follows: 


$$
x^{*}=b_{0}^{D}\left[1-\delta\left(\frac{n-1-q}{n-1}\right)\right]
$$

As $(n-1-q) /(n-1)>0, \delta>0$ and $b_{0}{ }^{D}>0$, it follows straightforwardly that:

Remark 1: $x^{*}<b_{0}{ }^{D}$

Thus, $A$ can propose less than the default payoff in period 1 to each $j \in M_{l}$ while still inducing each to vote 'yes'.

The 'incremental budgeting' strategies can be defined more formally as follows (where $j$ 's strategy of voting 'yes' to period-1 allocations greater than $x *$ is denoted $\sigma_{j}^{I}$ ):

$\sigma_{A}{ }^{I}:$ In period 1 , choose at $\operatorname{random}^{15}$ a subset of $J$, denoted $M_{1}$, where card $\left(M_{1}\right)=q$, and propose $b_{j}^{P l}$

$$
\begin{aligned}
& =x^{*} \quad \text { if } j \in M_{1} \\
& =0 \quad \text { if } j \notin M_{l} \\
& =R-q x^{*} \quad \text { if } j=A
\end{aligned}
$$

If $\mathbf{b}^{P 1}$ passes (i.e. $\mathbf{b}^{L 1}=\mathbf{b}^{P 1}$ ), then, in period 2, propose $b_{j}^{P 2}$

$$
\begin{aligned}
& =b_{0}{ }^{D} \quad \text { if } j \in M_{1} \\
& =0 \quad \text { if } j \notin M_{1} \\
& =R+I-q b_{0}{ }^{D} \text { if } j=A
\end{aligned}
$$

If $\mathbf{b}^{P 1}$ does not pass, then, in period 2, choose at random a subset of $J$, denoted $M_{2}$, where card $M_{2}=q$, and propose $b_{j}^{P 2}$

$$
\begin{array}{ll}
=b_{0}{ }^{D} & \text { if } j \in M_{2} \\
=0 & \text { if } j \notin M_{2} \\
=R+I-q b_{0}{ }^{D} & \text { if } j=A
\end{array}
$$

$\sigma_{j}^{I}: \quad$ In period 1 , if $b_{j}^{P 1} \geq x^{*}$, then vote 'yes'; otherwise, vote 'no'. In period 2 , if $b_{j}^{P 2} \geq b_{0}{ }^{D}$, then vote 'yes'; otherwise, vote 'no'.

It can be shown that the strategies defined above are mutual best responses; this will be termed the 'incremental budgeting' equilibrium:

\footnotetext{
${ }^{15}$ The random choice of the $q$ legislators means that $A$ is, in a sense, following a mixed strategy. More precisely, $A$ 's strategy involves the (random) choice of coalition members, followed by the proposal of a budget that includes offers of allocations to these members. This formulation is preferable to one that characterizes $A$ 's strategy as a probabilistic offer to each $j$ (i.e. $A$ offers $b_{0}{ }^{D}$ to each $j$ with probability $\left.(q /(n-1))\right)$ because it avoids the possibility of realizations of $A$ 's strategy that lead to the defeat of $A$ 's proposal in equilibrium. Myerson (1993) adopts the latter approach, but in a very different context, with multicandidate elections and an infinite number of voters.
} 
Proposition 1: Consider the 2-period budgeting game. There exist subgame perfect 'incremental budgeting' equilibria, where $A$ plays $\sigma_{A}^{I}$ and each $j$ plays $\sigma_{j}^{I}$. In an equilibrium of this type, $A$ chooses $q j$ 's at random to form $M_{1}$, and proposes $x^{*}$ for each $j \in M_{1} ; \mathbf{b}^{L I}=\mathbf{b}^{P I}$. In period 2, $A$ proposes $b_{0}{ }^{D}$ for each $j \in M_{1} ; \mathbf{b}^{L 2}=\mathbf{b}^{P 2}$.

Proof: To show that $\sigma_{A}^{I}$ and $\sigma_{j}^{I}$ are mutual best responses:

Suppose that $A$ plays $\sigma_{A}{ }^{I}$. Consider $j \in M_{l}$; deviating by voting 'no' in period 1 will lead to the defeat of $\mathbf{b}^{P I}$ (as each $j$ is pivotal) and lead to an expected payoff of $x^{*}$. Thus, this deviation does not raise $j$ 's payoff. Deviating by voting 'no' in period 2 leads to the default payoff $b_{0}{ }^{D}$ in period 2, which is identical to the equilibrium payoff in period 2; thus, $j$ cannot raise her payoff by deviating. For $j \notin M_{1}$, deviating by voting 'yes' in either period clearly cannot raise her payoff above 0 .

Suppose that each $j$ plays $\sigma_{j}^{I}$. If $A$ deviates by offering $b_{j}^{P I}<x^{*}$ to any $j \in M_{l}, \mathbf{b}^{P I}$ will fail, and $A$ will receive a payoff of $b_{A}{ }^{D}$ in period 1 , which is strictly less than the equilibrium period-1 payoff $R-q x^{*}$ (recall Equation (1a) and A1, while $q<(n-1)$ by assumption, and $x^{*}<b_{0}{ }^{D}$ by Remark 1), while the period-2 payoff is unchanged. Deviating by offering $b_{j}^{P I}>x^{*}$ to any $j \in M_{1}$ also makes $A$ worse off. Similar arguments hold for period 2. Moreover, note that $A$ has no incentive to deviate by changing the membership of the coalition across periods (i.e. setting $M_{2} \neq M_{1}$ ). Regardless of the identity of the $q$ legislators in $M_{2}$, $A$ 's payoff in period 2 is $R+I-q b_{0}{ }^{D}$. Of course, there is no incentive for $A$ to expand the coalition to include more than $q$ members. End of Proof.

This incremental equilibrium is not unique, however. Indeed, there is a continuum of equilibria. To see this, note that $A$ 's strategy (with respect to the choice of $M_{2}$ ) can be characterized by a probability distribution over the elements of the set $J$, indicating the probability that each $j$ will be included in the period 2 coalition (e.g. assigning probability $p_{j}$ to $j$ being chosen, contingent on $\mathbf{b}^{P 1}$ passing). Each probability distribution defines an allocation $x_{j}{ }^{*}$ that makes $j$ indifferent between voting 'yes' and 'no' in period 1. Consider a strategy for $A$ that involves choosing $q j$ 's in period 1 , and proposing $x_{j}^{*}$ to each $j \in M_{i}$; if $\mathbf{b}^{P 1}$ passes, $A$ includes each of these $j$ 's in $M_{2}$ with probability $p_{j}$. This strategy and a strategy for $j$ that involves voting 'yes' in period 1 whenever $b_{j}^{P 1}>x_{j}{ }^{*}$ clearly constitute mutual best responses. There are, however, an infinite number of possible probability distributions over the $j$ 's; thus, there are an infinite number of equilibria (note that the equilibrium characterized in Proposition 1 involves setting $p_{j}=1$ for $j \in M_{l}$ and $p_{j}=0$ for $j \notin M_{l}$ ).

In comparing the properties of the incremental budgeting equilibria in Proposition 1 with those of the other types of equilibria, it is helpful to focus on an extreme case that will be labeled 
the 'nonincremental budgeting' equilibrium. This involves $A$ randomizing completely over the $j$ 's (i.e. setting the $p_{j}$ 's equal) in period 2 , even when $\mathbf{b}^{P 1}$ passes. The 'nonincremental' strategies (denoted $\sigma_{A}^{N}$ and $\sigma_{j}^{N}$ ) can be defined as follows:

$\sigma_{A}{ }^{N}:$ In period 1 , choose at random a subset of $J$, denoted $M_{l}$, where card $\left(M_{1}\right)=q$, and propose $b_{j}^{P l}$

$$
\begin{aligned}
& =b_{0}{ }^{D} \quad \text { if } j \in M_{1} \\
& =0 \quad \text { if } j \notin M_{1} \\
& =R-q b_{0}{ }^{D} \quad \text { if } j=A
\end{aligned}
$$

In period 2, choose at random a subset of $J$, denoted $M_{2}$, where card $\left(M_{2}\right)=q$, and propose $b_{j}^{P 2}$

$$
\begin{array}{ll}
=b_{0}{ }^{D} & \text { if } j \in M_{2} \\
=0 & \text { if } j \notin M_{2} \\
=R+I-q b_{0}{ }^{D} & \text { if } j=A
\end{array}
$$

$\sigma_{j}^{N}: \quad$ In each period $i=1,2$, if $b_{j}^{P i} \geq b_{0}{ }^{D}$, then vote 'yes'; otherwise, vote 'no'.

It can be shown that:

Proposition 2: Consider the 2-period budgeting game. There exist subgame perfect 'nonincremental budgeting' equilibria, where $A$ plays $\sigma_{A}{ }^{N}$ and each $j$ plays $\sigma_{j}^{N}$. In an equilibrium of this type, $A$ chooses $q j$ 's at random to form $M_{1}$, and proposes $b_{0}{ }^{D}$ for each $j \in M_{1} ; \mathbf{b}^{L 1}=\mathbf{b}^{P 1}$. In period 2, $A$ chooses $q j$ 's at random to form $M_{2}$, and proposes $b_{0}{ }^{D}$ for each $j \in M_{1} ; \mathbf{b}^{L 2}=\mathbf{b}^{P 2}$.

Proof: To show that $\sigma_{A}{ }^{N}$ and $\sigma_{j}^{N}$ are mutual best responses:

Suppose that $A$ plays $\sigma_{A}{ }^{N}$. Consider a $j$ who is offered an allocation of $b_{0}{ }^{D}$ in either period. Deviating by voting 'no' entails the proposal's defeat, and leads to the default payoff $b_{0}{ }^{D}$; thus, $j$ cannot raise her payoff by defecting when she is included in $A$ 's coalition. Similarly, a $j$ who is offered 0 cannot raise her payoff by deviating and voting 'yes'.

Suppose that each $j$ plays $\sigma_{j}^{N}$. If $A$ deviates (in either period) by offering $b_{j}^{P i}<b_{0}{ }^{D}$ to any $j$ in the coalition, the proposal will be defeated; $A$ will receive the default payoff $b_{0}{ }^{D}$, rather than the equilibrium payoff $R-q b_{0}{ }^{D}$ (period 1) or $R+I-q b_{0}{ }^{D}$ (period 2). Note that, by Equation (1) and A1, and given that $q<(n-1)$ by assumption, it follows that $R-q b_{0}{ }^{D}>$ $b_{0}{ }^{D}$, and (as $\left.I \geq 0\right), R+I-q b_{0}{ }^{D}>b_{0}{ }^{D}$. Thus, $A$ cannot raise her payoff by deviating in this way. Deviating by proposing $b_{j}^{P i}>b_{0}{ }^{D}$ also leads to a strictly lower payoff. $A$ also does not have an incentive to deviate by changing the selection of $M_{2}$ from the random process specified in $\sigma_{A}{ }^{N}$ : any coalition of $q j$ 's leads to the same payoff in period 2 (of $R+I-$ $q b_{0}{ }^{D}$ ), so the random choice of $M_{2}$ is a best response. End of Proof.

This equilibrium replicates in each period the outcome of the 1-period game (discussed above). 


\section{3) Comparing Incremental and Nonincremental Equilibria}

A noteworthy feature of these different equilibria (in Propositions 1 and 2) is that they lead to systematically different payoffs for $A$ and the $j$ 's. More precisely, let $U_{A}{ }^{I}\left(\sigma_{A}{ }^{I}, \sigma_{j}{ }^{I}\right)$ and $U_{A}^{N}\left(\sigma_{A}{ }^{N}, \sigma_{j}^{N}\right)$ be $A$ 's payoffs in the incremental and nonincremental equilibria, respectively. Similarly, define $U_{j}^{I}\left(\sigma_{A}{ }^{I}, \sigma_{j}^{I}\right)$ and $U_{j}^{N}\left(\sigma_{A}{ }^{N}, \sigma_{j}^{N}\right)$ for $j=2, \ldots, n$ as the expected payoff of each $j$ in the incremental and nonincremental equilibria. Then:

Proposition 3: $U_{A}{ }^{I}>U_{A}{ }^{N}$ and $U_{j}^{I}<U_{j}^{N}$ for $j=2, \ldots, n$. Moreover, $U_{A}{ }^{I}$ is the highest payoff that $A$ can obtain in any subgame perfect equilibrium.

Proof: $U_{A}^{I}\left(\sigma_{A}^{I}, \sigma_{j}^{I}\right)=\left(R-q x^{*}\right)+\delta\left(R+I-q b_{0}{ }^{D}\right)$ and

$U_{A}^{N}\left(\sigma_{A}^{N}, \sigma_{j}^{N}\right)=\left(R-q b_{0}^{D}\right)+\delta\left(R+I-q b_{0}^{D}\right)$

As $x *<b_{0}{ }^{D}$ (see Remark 1), it follows that $U_{A}{ }^{I}>U_{A}{ }^{N}$

$U_{j}^{I}\left(\sigma_{A}^{I}, \sigma_{j}^{I}\right)=(q /(n-1))\left(x^{*}+\delta b_{0}^{D}\right)$

$U_{j}^{N}\left(\sigma_{A}{ }^{N}, \sigma_{j}^{N}\right)=(1+\delta)(q /(n-1)) b_{0}{ }^{D}$

Again, from Remark 1, it follows that $U_{j}^{I}<U_{j}^{N}$ for $j=2, \ldots, n$

Finally, recall the partially incremental equilibria, where there is some probability $(<1)$ that $j \in M_{1}$ is included in the period-2 coalition. Clearly, any reduction in this probability below 1 requires that $A$ propose a larger period-1 allocation $x_{j}^{*}$ to compensate. Consequently, $A$ 's period-1 payoff must be lower than $R-q x^{*}$; on the other hand, $A$ 's period-2 payoff is always $R+I-q b_{0}{ }^{D}$. Thus, $U_{A}{ }^{I}$ is the highest payoff $A$ can obtain in any subgame perfect equilibrium. End of Proof.

The significance of this result is that the higher payoff received by $A$ under incremental budgeting may provide a rationale for why traditional or 'classical' budgeting was organized in this fashion. More specifically, it suggests that those legislators with agenda setting power on budgetary policy may wish to foster an incrementalist ethos in order to increase the payoffs they receive from budget allocations.

\section{4) Solution with Commitment}

The analysis of the 2-period game above proceeded under the assumption that $A$ cannot make commitments in period 1 to allocate money in particular ways in period 2. However, it may be the case that, in many real-world budgeting contexts, there exist institutional mechanisms that enable $A$ to make such a commitment (at least to some degree). This section analyzes the 2period model under the assumption that $A$ can make the following commitment to legislator $j$ $(j=2, \ldots, n)$ : 
Definition 1 ('Grandfathering'): If $b_{j}^{P 1}=x$, and $\mathbf{b}^{P 1}$ is enacted $\left(\mathbf{b}^{L I}=\mathbf{b}^{P 1}\right)$, then $b_{j}^{P 2} \geq x \forall j \in J$

This amounts to a commitment to 'grandfather' budget allocations, contingent on the proposed period-1 budget being enacted. Institutions that may enable $A$ to commit to grandfathering include budget baselines and procedural rules that preclude (or impose high transactions costs on) revisiting past allocations (as discussed in Section 1).

The main result of this section is that, if $A$ can commit to grandfathering, then the nonincremental (and partially incremental) equilibria are eliminated:

Proposition 4: Consider the 2-period budgeting game, and assume that $A$ can commit to grandfathering budget allocations (as specified in Definition 1). Then, the only subgame perfect equilibria involve incremental budgeting (i.e. the same equilibrium outcomes as when $A$ plays $\sigma_{A}{ }^{I}$ and each $j$ plays $\sigma_{j}^{I}$ ).

Proof: Consider the subgame consisting of period 2, and suppose that $\mathbf{b}^{P 1}$ has been enacted. $A$ will always choose $M_{2}$ such that card $\left(M_{2}\right)=q$. The cost incurred by $A$ in passing the period-2 budget can be expressed as:

$$
q b_{0}^{D}+\sum_{j \in M_{1} \text { and } j \notin M_{2}} b_{j}^{P 1}
$$

i.e. $A$ needs to allocate $b_{0}{ }^{D}$ to each of the $q$ members of $M_{2}$. In addition, if there are any $j$ 's who were part of $M_{1}$ but are not included in $M_{2}, A$ must allocate each of these $j$ 's her period-1 allocation $b_{j}^{P l}$ in order to satisfy the commitment to grandfathering. Clearly, this cost can be minimized by ensuring that there is no $j \in M_{1}$ who is excluded from $M_{2}$ (note that this is feasible, as card $\left(M_{1}\right)=$ card $\left.\left(M_{2}\right)=q\right)$. Thus, setting $M_{2}=M_{1}$ is strictly preferred by $A$ to any alternative strategy (as it leads to a period-2 payoff of $R+I-q b_{0}^{D}$, which is strictly higher than that resulting from any difference between $M_{2}$ and $M_{1}$ ). Therefore, any subgame perfect equilibrium must involve $M_{2}=M_{1}$; i.e. incremental budgeting. Given this, $A$ 's optimal period-1 strategy is to propose $x^{*}$ for each $j \in M_{l}$ (as in the strategy $\sigma_{A}{ }^{I}$ ). As shown in Proposition 1, each $j$ 's best response is $\sigma_{j}^{I}$; moreover, any best response must involve the same action (i.e. voting 'yes') in period 1. Thus, the equilibrium outcomes are those specified in Proposition 1. End of Proof.

Thus, if $A$ has the ability to precommit to grandfathering period-1 allocations, incremental budgeting represents the unique subgame perfect equilibrium (up to the choice of $M_{1}$ ); in effect, $A$ can ensure that she receives the higher payoff associated with the incremental budgeting outcome.

\section{5) A Note on Credible Threats of Exclusion}

In deriving the results above, it was assumed that, in the event that $\mathbf{b}^{P 1}$ is defeated, $A$ simply randomizes over all legislators in choosing $M_{2}$. If it is assumed that it is possible for $A$ to credibly make more specific threats to the members of $M_{1}$ off the equilibrium path, then the 
results are strengthened. In particular, suppose that $A$ can threaten to exclude as many members of $M_{1}$ who vote 'No' as possible from $M_{2}$. Thus, if the $q$ legislators in $M_{1}$ vote 'No' in period $1,{ }^{16}$ then, when building a period 2 coalition, $A$ will turn first to the $(n-1-q)$ legislators who were not part of $M_{l}$, recruiting only the other $(2 q-n+1)$ from the members of $M_{l}$. The probability that a legislator who votes 'No' in period 1 is subsequently included in $M_{2}$ is reduced to $(2 q-n+1) / q$. The analog to $x^{*}$ under these circumstances, denoted $x^{* *}$, can be defined as:

$$
x * *=b_{0}^{D}\left[1-\delta\left(\frac{n-1-q}{q}\right)\right]
$$

As $(n-1)>q, \delta>0$ and $b_{0}{ }^{D}>0$ by assumption, it follows that $(n-1-q) /(n-1)<(n-1-q) / q$, and hence that:

Remark 2: $x^{* *}<x^{*}$

That is, allowing $A$ to make a credible out-of-equilibrium threat of exclusion from the period 2 coalition further lowers the period 1 allocation necessary to ensure passage of $\mathbf{b}^{P 1}$, thereby raising $A$ 's payoff and reducing the expected payoff of each junior legislator.

An interesting special case occurs when the legislature operates under a simple majority rule, so that $q=(n-1) / 2$. Then, it is possible for $A$ to exclude every single member of $M_{1}$ if she votes 'No' in period 1, so the threat involves a zero probability of being in $M_{2}$. In these circumstances, $x^{* *}=(1-\delta) b_{0}{ }^{D}$; intuitively, $x^{* *}$ only needs to be sufficient to compensate for discounting, as $j \in M_{1}$ faces a choice between receiving $b_{0}{ }^{D}$ now and 0 next period (by voting 'no') or $x^{* *}$ now and $b_{0}{ }^{D}$ next period (by voting 'yes').

\section{6) A Note on Endogenous Default Payoffs}

In the preceding analysis, it has been assumed (as discussed in Section 3.1) that the default payoffs of legislators are exogenous: they are independent of the realized pattern of play in thegame. As noted earlier, this rules out the case where $b_{j}^{D 2}=b_{j}^{L 1}$ (i.e. legislator $j$ 's period 2 default is her period 1 allocation). To see why this assumption may seem restrictive, suppose that $A$ is unable to commit to grandfathering (in the sense of Definition 1). Suppose further that $A$ offers some amount $x$ (sufficient to induce a vote for the proposal) to $q j$ 's in period 1 . This proposed allocation is enacted, by assumption. Then, in period 2 , each $j \in M_{l}$ has default payoff

\footnotetext{
${ }^{16}$ It is assumed here that all the members of $M_{l}$ vote 'No', given the assumption of symmetric behavior. If only some of them vote 'No', then $A$ 's threat to exclude is even more potent, as the probability of a legislator who voted 'No' being part of $M_{2}$ can be reduced even further.
} 
$x$, while each $j \notin M_{l}$ has default payoff 0 . Obviously, the latter will be the cheapest to buy, from $A$ 's point of view; thus, $A$ will build a coalition $M_{2}$ that contains as many $j \notin M_{l}$ as possible, and each of these $j$ 's will be offered an allocation of 0 . Only as many $j \in M_{l}$ as absolutely necessary to ensure passage will be included in $M_{2}$, with each of these $j$ 's being offered $x$ (in the special case of a simple majority rule, no $j \in M_{1}$ will be included in $M_{2}$ ). This result would seem to be the opposite of incremental budgeting - those legislators excluded from the period 1 coalition are always included in period 2, while those in the period 1 coalition are only included in period 2 if absolutely necessary.

However, if it is possible for $A$ to comit to grandfathering (as defined above), then incremental budgeting outcomes are possible, even when $b_{j}^{D 2}=b_{j}^{L 1}$. To see this, consider a simple example. Let $n=5$, with $L=\{A, 2,3,4,5\}, q=2$ (i.e. a simple majority rule), and $\delta=1$. Suppose that $b_{j}^{D 1}=b_{0}{ }^{D}$ (i.e. the first-period default is exogenous) and $b_{j}^{D 2}=b_{j}^{L 1}$, and that $M_{l}=$ $\{2,3\}$; in addition, suppose that (as in Section 3.5) $A$ can credibly threaten to exclude $j$ 's from the period 2 coalition. In the absence of commitment to grandfathering, the equilibrium involves nonincremental budgeting, with $A$ offering $b_{0}{ }^{D}$ to 2 and 3 in period 1, and 0 to all $j$ 's in period 2, with $M_{2}=\{4,5\} ; A$ 's payoff is $2 R+I-2 b_{0}{ }^{D}$. Now suppose that commitment to grandfathering (in the sense of Definition 1) is possible. The equilibrium here involves $A$ offering $b_{0}{ }^{D} / 2$ to 2 and 3 in each period. Of course, 4 and 5 are technically 'included' in $M_{2}$, in that they are offered their default of 0 , and will vote 'yes'; however, when there is at least a small $\varepsilon$ cost of expanding the coalition, the $M_{1}=M_{2}$ feature of incrementalism will hold. This equilibrium gives $A$ the same payoff $2 R+I-2 b_{0}{ }^{D}$ as that from nonincremental budgeting (where $A$ does not precommit to grandfathering, and changes the coalition from one period to the next). Thus, incremental budgeting can be an optimal strategy for $A$ when commitment to grandfathering is available, even if the default payoffs of junior legislators are endogenous.

\section{4) Incremental Budgeting with an Infinite Horizon}

The basic model developed in Section 3 assumed that the budgeting game is played only over two periods. This section extends this framework to budgeting with an infinite horizon; the budgeting game (involving a budget proposal by $A$ and a vote under a closed rule by the legislature) is assumed to be repeated infinitely. The rationale for this assumption is that there is uncertainty regarding how long $A$ 's proposal power will last. Of course, as incremental 
budgeting outcomes can be sustained in the 2-period case (with and without commitment to grandfathering), it is obvious that they can also be sustained in the infinitely repeated game. The aim of this section is thus not to establish the existence of incremental outcomes, but rather to analyze how the infinite horizon setting affects the gains to $A$ from incremental budgeting. As in Section (3.4), it is assumed here that $A$ can credibly precommit to the grandfathering of budget allocations. Incremental budgeting outcomes can of course be sustained even in the absence of such commitment (though, in those circumstances, there are many other types of equilibria). This section also returns to the assumption that $A$ randomizes with equal probability over all $j$ 's if a proposal is defeated.

The (exogenous) stream of revenues available to the legislature for budgetary allocations will be denoted by $R_{t}, t=1,2, \ldots$, where $R_{t} \geq(n-1) b_{0}{ }^{D}+b_{A}{ }^{D} \forall t .{ }^{17}$ In the 2-period model, the 'last-period' factor in period 2 suggested that the default payoff for $A$ in the last period should differ from that in period 1 . Here, however, the game to be played in the future is identical at each point in time; thus, there is a common default payoff $b_{A}{ }^{D}$. It is assumed that the infinite sequence represented by the (discounted) revenue stream converges - i.e. that the discounted sum of the $R_{t}$ 's is finite:

Condition 1: $\delta<1$ and $R^{*} \equiv \sum_{t=1}^{\infty} \delta^{t-1} R_{t}$ is finite

This condition is assumed to hold throughout the analysis in this section. The game in the infinitely repeated case is a straightforward extension of that described in Section 3. In each period, $A$ proposes a feasible budget allocation, ${ }^{18}$ and the legislature votes the proposal up or down, with the default allocations being given by $b_{0}{ }^{D}$. The definition of 'grandfathering' is now somewhat more general than in Section 3:

Definition 1'('Grandfathering'): If $b_{j}^{P t}=x$, and $\mathbf{b}^{P k}$ is enacted $(\forall k \geq t)$, then $b_{j}^{P(k+1)} \geq x \forall j \in J$

That is, if $A$ offers $x$ to $j$ in period $t$, and the proposal passes, then $A$ (credibly) commits to offering at least $x$ to $j$ as long as the previous period's proposal has passed. This is a straightforward generalization of Definition 1.

\footnotetext{
${ }^{17}$ Note that it is not necessary to impose the restriction that $R_{t+1} \geq R_{t} \forall t$ (i.e. that the revenue stream is nondecreasing) to derive the results below.

18 'Feasibility' here refers to the budget constraint. Note that, as in the previous analysis, a period-by-period constraint is being assumed. However, the results would not change fundamentally if $A$ were permitted to reallocate revenue across different periods, as long as there are some constraints on borrowing (so that, for instance, $A$ cannot spend the entire revenue stream in $t=1$, and allocate $\mathbf{0}$ thereafter).
} 
As before, the solution concept involves looking for subgame perfect Nash equilibria. ${ }^{19}$ The central result of this section is:

Proposition 5: Suppose that Condition 1 holds, and that precommitment to grandfathering is possible; then, in every subgame perfect equilibrium of the infinitely repeated game,

(i) $\quad A$ chooses $M_{l}$ (consisting of $q$ junior legislators) randomly in period 1

(ii) $\forall t=1,2, \ldots, A$ proposes $b_{j}^{P t}$

$$
\begin{array}{ll}
=\gamma b_{0}{ }^{D} & \text { if } j \in M_{1} \\
=0 & \text { if } j \notin M_{1}
\end{array}
$$

where

$$
\gamma=\frac{1-\delta}{1-\frac{\delta q}{n-1}}
$$

(note that $\gamma<1$ for any $\delta>0$ ), and $\mathbf{b}^{P t}$ passes $\forall t=1,2, \ldots$

(iii) $A$ 's equilibrium payoff (in present value terms) is

$$
R *-\frac{(n-1) q b_{0}^{D}}{n-1-\delta q}
$$

(which exceeds $A$ 's default payoff $b_{A}{ }^{D} /(1-\delta)$ )

(iv) $j$ 's expected payoff (in present value terms) is

$$
q b_{0}{ }^{D} /(n-1-\delta q)
$$

Proof: (i) The MWC result from the 2-period model extends to this context, so $M_{l}$ will consist of $q j$ 's; $A$ will choose them randomly because the $j$ 's are identical.

(ii) Let $b_{j}^{P 1}=x \forall j \in M_{1}$. Consider period $t$. There are two relevant kinds of histories to consider. Following histories in which $\mathbf{b}^{P(t-1)}$ failed, the subgame following this history is identical to the entire game from $t=1$; thus, in period $t, A$ will choose $M_{t}$ randomly, and offer $b_{j}^{P t}=x \forall j \in M_{t}$. Following histories in which $\mathbf{b}^{P(t-1)}$ passed, note that there must have been a continuous history of passage since period $h$, where $1 \leq h \leq t-1$. By Definition 1' above, it follows that $A$ chose a coalition consisting of $q j$ 's, $M_{h}$, in period $h$, and that $A$ offers $b_{j}{ }^{P t}=x \forall j \in M_{h}$ in period $t$. (Note that, in each case, it will be optimal for $A$ to set $b_{j}^{P t}=0$ for $j$ 's outside the coalition)

To characterize $x$ : consider $t=1$, or $t>1$ following a history in which $\mathbf{b}^{P(t-1)}$ failed. As noted above, $A$ will choose $q j$ 's randomly to form $M_{t}$. Consider the choice faced by $j \in$

\footnotetext{
${ }^{19}$ In addition, an assumption of stationarity is made, requiring that behavior be identical in all subgames that are structurally essentially identical. A complication here is that the stage games may not be precisely identical, because the future revenue stream may differ in different periods. However, this does not fundamentally affect the results, as Condition 1 ensures that, at any point in time, the future revenue stream converges.
} 
$M_{i}$ : voting 'yes' will lead (given the definition of incrementalism) to a payoff of $x(1+\delta+$ $\left.\delta^{2}+\ldots\right)=x /(1-\delta)$. Voting 'no' leads to a payoff of $b_{0}{ }^{D}$ in period $t$, and to a $(q /(n-1))$ probability of being included in next period's coalition - i.e. to a payoff of $b_{0}{ }^{D}+(q /(n-$ 1)) $x\left(\delta+\delta^{2}+\ldots\right)=b_{0}{ }^{D}+(q /(n-1))(\delta /(1-\delta)) x$. Thus, $A$ must choose $x$ so as to just induce $j$ to vote 'yes':

Rearranging yields $x=\gamma b_{0}{ }^{D}$.

$$
x /(1-\delta)=b_{0}{ }^{D}+x q \delta /((n-1)(1-\delta))
$$

An alternative approach is to assume that deviation by $j$ involves voting 'no' whenever the opportunity arises (i.e. 'no' in period $t$, 'no' in period $(t+1)$ if $j$ is in $M_{t+1}$, which happens with probability $(q /(n-1))$, and so on). This leads to the same expression for $x$.

Given the above proposal, each $j \in M_{t}$ will vote 'yes' $\forall t$ (recall that $j$ votes 'yes' when indifferent); thus, $\mathbf{b}^{P t}$ passes $\forall t=1,2, \ldots$

To establish subgame perfection: consider the subgame beginning after period $t$. There are two types of histories to consider:

- after a history in which $\mathbf{b}^{P t}$ passes, note that $A$ is constrained by Definition $1^{\prime}$. Using the strategies specified above, $A$ will offer each $j \in M_{h}$ (where $h=1$ if $A$ 's proposals have all passed, or $1<h<t$ if one or more previous proposals have failed (where $h$ 1 is the last period where a proposal failed)) $x=\gamma b_{0}{ }^{D}$ in period $t$ and each subsequent period, and each $j \in M_{h}$ will vote 'yes' each period; from the reasoning above, this constitutes a Nash equilibrium

- after a history in which $\mathbf{b}^{P t}$ fails, $A$ will choose $M_{t+l}$ randomly, and offer $x=\gamma b_{0}{ }^{D}$ to each $j \in M_{t+1}$ in period $t$ and each subsequent period, and each $j \in M_{t+1}$ will vote 'yes' each period; from the reasoning above, this also constitutes a Nash equilibrium

This reasoning applies for each $t$; thus, the strategies specified in the Proposition are a Nash equilibrium in every subgame. This establishes subgame perfection. (Note that there are many subgame-perfect equilibria, corresponding to different choices of $M_{1}$; however, all have the characteristics specified in the Proposition).

(iii) Given the equilibrium strategies, $A$ allocates, in present value terms, $\gamma b_{0}{ }^{D}\left(1+\delta+\delta^{2}\right.$ $+\ldots$ ) to each of the $q$ members of the coalition $M_{1}$. A also allocates the remainder of the (finite) revenue stream $R^{*}$ to her own district; thus, her aggregate allocation is

$$
R^{*}-\gamma b_{0}{ }^{D}\left(1+\delta+\delta^{2}+\ldots\right)
$$

in present value terms. Substituting in the expression for $\gamma$ and simplifying gives the result.

To show that $A$ 's payoff exceeds her default payoff, note that the latter involves receiving $b_{A}{ }^{D}$ each period (a present value of $b_{A}{ }^{D} /(1-\delta)$ ). Consider the smallest revenue stream that is consistent with the assumptions of the model, namely, $R_{t}=(n-1) b_{0}{ }^{D}+b_{A}{ }^{D} \forall t$; then, $R^{*}=\left((n-1) b_{0}{ }^{D}+b_{A}{ }^{D}\right) /(1-\delta)$, and $A$ 's payoff can be expressed as: 


$$
\frac{b_{A}^{D}}{1-\delta}+(n-1) b_{0}^{D}\left(\frac{n-1-\delta q-(1-\delta)}{(1-\delta)(n-1-\delta q)}\right)
$$

Note that the expression in brackets is greater than 0 , so that $A$ 's payoff exceeds the default payoff $b_{A}{ }^{D} /(1-\delta)$. As this holds for the minimum possible revenue stream, it must also hold for any (finite) revenue stream. Thus, $A$ always prefers to play the strategy specified in the proposition, rather than receive the default payoff each period.

(iv) Each $j$ has a $q /(n-1)$ probability of being included in $M_{1}$. If so, she receives a stream of payoffs $\gamma b_{0}{ }^{D}\left(1+\delta+\delta^{2}+\ldots\right)$ with present value $\gamma b_{0}{ }^{D} /(1-\delta)$; otherwise, she receives 0 each period. Thus, her expected payoff, in present value terms, is

$$
q \gamma b_{0}{ }^{D} /[(n-1)(1-\delta)]
$$

Substituting in the expression for $\gamma$ and simplifying yields the result. End of Proof.

The result above generalizes Proposition 4 to the case where the budgeting game is repeated infinitely. It is noteworthy that this extension to an infinitely repeated game reinforces the result from the 2-period game. Specifically, $A$ can now offer a lower per-period allocation $\left(\gamma b_{0}{ }^{D}\right)$ to each member of the winning coalition than the $x^{*}$ defined in Equation (2) above, and still ensure passage of the budget:

Remark 3: $\gamma b_{0}{ }^{D}<x^{*}$

Proof: From Proposition 5(ii), $\gamma b_{0}{ }^{D}$ can be expressed as:

$$
\gamma b_{0}^{D}=\frac{(1-\delta)(n-1)^{2}}{(n-1)(n-1-\delta q)}
$$

and $x^{*}$ (from Equation (2)) can be expressed as:

$$
x^{*}=\frac{(n-1-\delta q)[(n-1)(1-\delta)+\delta q]}{(n-1)(n-1-\delta q)}
$$

The numerator of this expression can be simplified as follows:

$$
\begin{aligned}
& (1-\delta)(n-1)^{2}-\delta q(1-\delta)(n-1)+\delta q(n-1)-\delta^{2} q^{2} \\
= & (1-\delta)(n-1)^{2}+\delta^{2} q(n-1-q)
\end{aligned}
$$

As $q<n-1, q>0$, and $\delta>0$ by assumption, it follows that $\gamma b_{0}{ }^{D}<x^{*}$. End of Proof.

This result suggests that $A$ 's gains from incremental budgeting are even greater in the infinitely repeated context than in the simple 2-period setting of Section 3. Intuitively, the consequences for $j$ of being excluded from the coalition are more severe when the time horizon involved is longer, so those included in the coalition will be willing to accept a lower per-period payoff. 
Moreover, there is no final period in which each coalition member must be allocated her default payoff. Note that, because it is not necessary to impose a restriction that the revenue stream is nondecreasing, another significant implication is that a declining revenue stream does not, by itself, undermine the budget proposer's incentive to institute incrementalism.

The expression for $\gamma$ in Proposition 5(ii) also yields some (potentially) testable implications regarding how $A$ 's benefits from incrementalism change in response to the model's parameters. In particular, the higher is $\delta$, the lower is $\gamma$, the more patient are the legislators, the greater is the benefit to $A$ of incremental budgeting. This is because more patient $j$ 's will weight the loss from their possible noninclusion in future coalitions more heavily, and hence will be more willing to accept a lower per-period allocation in exchange for the certainty of inclusion. In addition, the larger is the number of votes required for passage (i.e. the higher is $q$ relative to $n$ ), the higher is $\gamma$. Thus, supermajority requirements for budgetary bills will, ceteris paribus, tend to lower the benefits to $A$ from incrementalism, because they reduce the risk to $j$ 's of being excluded from future coalitions (and, hence, make $j$ 's less willing to accept low per-period allocations).

Finally, it should be emphasized that, for reasonable parameter values, the magnitude of the effect in Proposition 5 is quite substantial. That is, the difference between the $\gamma b_{0}{ }^{D}$ allocated to each coalition member each period and the default payoff $b_{0}{ }^{D}$ is large. For example, consider the following values: $n=101, q=60$ (so that 61 votes are required for passage), and $\delta=0.9$. Then, $\gamma b_{0}{ }^{D}=(10 / 46) b_{0}{ }^{D} \approx 0.22 b_{0}{ }^{D}$. Thus, for these (reasonable) parameter values, the allocation that is sufficient to attract the support of each coalition member is only approximately a fifth of the default allocation that the member would receive.

\section{[Table 1 about here]}

Table 1 shows the values of $\gamma$ (the fraction of the default payoff required to induce a coalition member to vote 'yes') that result from various values of $n,(q+1)$, and $\delta$. In addition to the generally low values of $\gamma$, the table illustrates how $\gamma$ changes in response to the parameters. As $\delta$ rises (i.e. legislators become more patient), $\gamma$ falls, while, as $\delta$ decreases, $\gamma$ rises. As $q$ increases relative to $n$ (which makes it less likely that those $j$ 's who vote 'no' can be excluded from the future coalition), $\gamma$ rises. Conversely, as $q$ decreases relative to $n, \gamma$ falls. Overall, the calculations suggest that the magnitude of the effect of incremental budgeting is substantial. 


\section{5) Incremental Budgeting with Heterogeneous Legislators}

The analysis so far has relied on the assumption that the junior legislators are ex ante identical in all respects. This section returns to the simple 2-period game of Section 3, and considers the situation where legislators differ (strictly) in terms of the default payoffs they receive if $A$ 's proposal is defeated. Specifically, it is assumed that $j$ 's default payoff is $b_{j}^{D}$, where, without loss of generality, the $j$ 's are numbered such that $b_{2}{ }^{D}<b_{3}{ }^{D}<b_{4}{ }^{D} \ldots<b_{n}{ }^{D}$ (they will be referred to for convenience as $j_{2}, j_{3}, \ldots j_{n}$ ). As before, revenue is assumed to be sufficient to cover the aggregate default payoffs, so that:

$$
R \geq \sum_{j=A}^{n} b_{j}^{D}
$$

Then, one can define:

Definition 2: The cheapest MWC, denoted $M_{C}$, consists of $q$ junior legislators $\{2,3, \ldots,(q+$ 1)\} (so that $\Sigma b_{j}^{D}$ is minimized).

Initially, consider the 2-period game with no precommitment to grandfathering. In the subgame consisting of period 2 , it is immediately apparent that the equilibrium proposal must involve $b_{j}^{P 2}$

$$
\begin{array}{ll}
=b_{j}^{D} & \text { if } j \in M_{C} \\
=0 & \text { if } j \notin M_{C}
\end{array}
$$

In period 1, this equilibrium proposal is anticipated, so each $j \in M_{C}$ kows that she has probability 1 of being included in the period-2 coalition; consequently, no $j \in M_{C}$ will accept a proposal that allocates her less than her default $b_{j}^{D}$. Conversely, each $j \notin M_{C}$ knows that she has probability 0 of being included in the period-2 coalition, and will hence not accept a proposal that allocates her less than her default $b_{j}^{D}$. Clearly, it will be cheapest for $A$ to build a coalition $M_{1}=M_{C}$; thus, the period-1 proposal will be identical to the period-2 proposal specified above. $A$ 's equilibrium payoff is: ${ }^{20}$

$$
(1+\delta) R+\delta I-(1+\delta) \sum_{j=2}^{q+1} b_{j}^{D}
$$

\footnotetext{
${ }^{20}$ Note that, from Eq. (9) above, this equilibrium payoff always exceeds $A$ 's default payoff of receiving $b_{A}{ }^{D}$ each period (given that $I \geq 0$ and $q+1<n$ ).
} 
Thus, without commitment to grandfathering, $A$ builds the cheapest coalition $M_{C}$ each period. The issue considered here is whether, when precommitment to grandfathering is available, $A$ can achieve a higher payoff. The circumstances in which this is possible can perhaps best be introduced through a very simple example. Suppose that $n=5$, and that the $j$ 's have the following default payoffs:

$$
b_{2}{ }^{D}=9 ; b_{3}{ }^{D}=10 ; b_{4}{ }^{D}=11 ; b_{5}{ }^{D}=12
$$

Assume a simple majority rule, so that $q+1=3$ (i.e. $A$ needs 2 coalition partners for the passage of the budget), and that $\delta=1$. Let $C^{C}$ be the cost to $A$ of a strategy that involves building $M_{C}$ each period. As $M_{C}=\left\{j_{2}, j_{3}\right\}$, it follows that $C^{C}=38$. Note that, without commitment, this is $A$ 's equilibrium cost (yielding equilibrium payoff $2 R+I-38$ ).

Suppose that $A$ has the ability to precommit to grandfathering, and seeks to build a coalition consisting of $j_{4}$ and $j_{5}$. Thus, $A$ proposes some positive allocation to each of $j_{4}$ and $j_{5}$ in period 1. If one of them votes 'no' and $\mathbf{b}^{P I}$ fails, then $A$ will minimize period-2 costs by proposing $\mathbf{b}^{P 2}=(\cdot, 9,10,0,0) .{ }^{21}$ The issue, then, is what allocations will induce $j_{4}$ and $j_{5}$ to vote 'yes' in period 1. Suppose that $A$ proposes $b_{5}{ }^{P I}=1$; then, $j_{5}$ will anticipate that, in period 2 , it will be cheaper for $A$ to replace her with $j_{2}$, despite the fact that $A$ will still have to allocate $b_{5}^{P 2}=1$ to satisfy the grandfathering commitment. Thus, $j_{5}$ will vote 'no'. By this reasoning, it is clear that a proposal $b_{5}{ }^{P I}=3$ will just induce $j_{5}$ to vote 'yes', as it eliminates the incentive for $A$ to drop $j_{5}$ from the period-2 coalition. Similarly, $b_{4}^{P I}=2$ will induce $j_{4}$ to vote 'yes' in period 1 (note that, as we are considering deviations from a candidate equilibrium one at a time, it is the difference between $b_{j}^{D}$ and the default payoff of the cheapest alternative $j$ that matters here). If $A$ proposes $\mathbf{b}^{P 1}=(\cdot, 0,0,2,3)$ and $\mathbf{b}^{P 2}=(\cdot, 0,0,11,12)$, each proposal will pass, and $A$ incurs a total cost of 28 (i.e. a payoff of $2 R+I-28$ ). Clearly, $A$ can benefit from the ability to precommit.

Now suppose that $q+1=4$, so that $A$ needs 3 coalition partners. Without commitment, the coalition would consist each period of $M_{C}=\left\{j_{2}, j_{3}, j_{4}\right\}$, with $C^{C}=60$. Assume that $A$ can precommit. Note that $A$ can no longer recruit the entire coalition from outside $M_{C}$. Instead, $A$ will build a coalition of all $j$ 's outside $M_{C}$, together with as many $j$ 's (specifically, the cheapest ones) from $M_{C}$ as required. The $j$ whose default determines how much each $j \notin M_{C}$ must be paid in

\footnotetext{
${ }^{21}$ This notation omits $A$ 's own allocation for convenience, to focus on the proposals for the $j$ 's.
} 
period 1 is now the cheapest $j \in M_{C}$ outside $A$ 's coalition. Thus, suppose $A$ proposes $\mathbf{b}^{P 1}=(\cdot, 9$, $10,0,1)$ and $\mathbf{b}^{P 2}=(\cdot, 9,10,0,12)$; then, each proposal will pass, and $A$ 's cost will be 51 .

To generalize from these examples, return to the more abstract formulation of the situation at the beginning of this section. Recall that the $j$ 's are numbered such that $b_{2}{ }^{D}<b_{3}{ }^{D}<b_{4}{ }^{D}$ $\ldots<b_{n}{ }^{D}$, and that $M_{C}=\left\{j_{2}, j_{3}, \ldots, j_{q+1}\right\}$, where card $M_{C}=q$. Legislators $\left\{j_{q+2}, j_{q+3}, \ldots, j_{n}\right\}$ are outside $M_{C}$, with card $J \sim M_{C}=n-q-1$. In the examples above, a strategy of choosing $M_{C}$ each period was contrasted with one that used $A$ 's precommitment capacity to lower the cost of the coalition. Strictly speaking, the outcomes associated with both these strategies appear 'incremental' (in the sense that the same coalition is maintained from period 1 to period 2). However, choosing $M_{C}$ each period is only incidentally incremental (in that it is simply equivalent to period-by-period optimization by $A$ ), whereas the other strategy relies crucially on the grandfathering commitment. Thus, the latter will be labeled an 'incremental' strategy in the analysis to follow, and the cost associated with adopting this strategy will be denoted by $C^{I}$.

The incremental strategy for $A$ involves including each $j \in J \sim M_{C}$ in the coalition. This secures the support of $(n-q-1)$ legislators, leaving a shortfall of $(2 q+1-n)$; this is made up by also including $\left\{j_{2}, j_{3}, \ldots, j_{2 q+2-n}\right\}$ in the coalition. The cost of securing passage of each period's budget using this strategy is:

$$
C^{I}=(1+\delta) \sum_{j=2}^{2 q+2-n} b_{j}^{D}+\sum_{j=q+2}^{n}\left(b_{j}^{D}-b_{2 q+3-n}^{D}\right)+\delta \sum_{j=q+2}^{n} b_{j}^{D}
$$

whereas the cost of simply choosing $M_{C}$ each period is:

$$
C^{C}=(1+\delta) \sum_{j=2}^{q+1} b_{j}^{D}
$$

It can be shown that:

Proposition 6: Consider the 2-period budgeting game with commitment, and suppose that legislators have strictly heterogeneous default payoffs, such that $b_{2}{ }^{D}<b_{3}{ }^{D}<b_{4}{ }^{D} \ldots<b_{n}{ }^{D}$. Then, if $\left(b_{n}{ }^{D}-b_{2}{ }^{D}\right)>0$ is sufficiently small, $C^{I}<C^{C}$ (i.e. the cost to $A$ of an incremental strategy is lower than that of choosing $M_{C}$ each period). 
Proof: Define $\varepsilon_{3}, \varepsilon_{4}, \ldots, \varepsilon_{n}$, where $\varepsilon_{j}>0 \forall j=3, \ldots, n$ and $\varepsilon_{j+1}>\varepsilon_{j} \forall j=3, \ldots, n-1$, such that:

$$
b_{j}^{D}=b_{2}^{D}+\varepsilon_{j}
$$

Then, it is possible to express $C^{I}$ and $C^{C}$ as:

$$
\begin{aligned}
C^{I}= & (1+\delta)(2 q+1-n) b_{2}^{D}+(1+\delta) \sum_{j=2}^{2 q+2-n} \varepsilon_{j} \\
& +\sum_{j=q+2}^{n} \varepsilon_{j}-(n-q-1) \varepsilon_{2 q+3-n}+\delta(n-q-1) b_{q+2}^{D}+\delta \sum_{j=q+2}^{n} \varepsilon_{j}
\end{aligned}
$$

Note that:

$$
\lim _{\varepsilon_{j} \rightarrow 0 \forall j} C^{I}=q+(1+\delta) q+1-n
$$

and

$$
\begin{array}{r}
\lim _{\varepsilon_{j} \rightarrow 0 \forall j} C^{C}=(1+\delta) q \\
C^{C}=(1+\delta) q b_{2}^{D}+(1+\delta) \sum_{j=2}^{q+1} \varepsilon_{j}
\end{array}
$$

Recall that $q<n-1$. Thus, when $\varepsilon_{j}=0 \forall j=3, \ldots, n, C^{I}<C^{C}$. Note that this is a strict inequality, Thus, there must exist a sequence of sufficiently small $\varepsilon_{j}$ 's, where $\varepsilon_{j}>0$ $\forall j=3, \ldots, n$ (and hence $\left(b_{n}{ }^{D}-b_{2}{ }^{D}\right)>0$ ) for which $C^{I}<C^{C}$. End of Proof.

This result shows that, if the heterogeneity among $j$ 's is not too great, then an incremental strategy will be optimal for $A$. To see the intuition for why this does not hold more generally, recall the simple example above, with $n=5, q+1=3$, and $\delta=1$. Suppose that the default payoffs are:

$$
b_{2}{ }^{D}=1 ; b_{3}{ }^{D}=2 ; b_{4}{ }^{D}=20 ; b_{5}{ }^{D}=21
$$

To secure passage, $A$ would have to propose $\mathbf{b}^{P 1}=(\cdot, 0,0,19,20)$ and $\mathbf{b}^{P 2}=(\cdot, 0,0,20,21)$, entailing a cost of $C^{I}=80$, whereas $C^{C}=6$. Clearly, if the legislators are sufficiently heterogeneous, $A$ cannot gain by adopting an incremental strategy. However, the general lesson of this section is that, even when there is some heterogeneity among the $j$ 's, there exist circumstances in which incrementalism is optimal.

\section{6) Conclusion, and Possible Extensions}

This paper has developed a formal analysis of the concept of incremental budgeting, linking the substantive concerns of public budgeting scholars with the theoretical approach of the 
rational choice tradition. A simple 2-period model was presented in Section 3, using a legislative bargaining framework in which an exogenously chosen agenda setter proposes budget allocations for each period. It was shown that the agenda setter can lower the cost of building a winning coalition, and thereby increase her payoff, through the use of incremental budgeting. In Section 4 , the model was extended to an infinitely repeated context, and it was shown that the basic result is reinforced in this setting. Moreover, calculations using reasonable values of the model's parameters suggest that the magnitude of the effect is quite large. Another significant implication is that a declining revenue stream does not, by itself, undermine the budget proposer's incentive to institute incrementalist practices. A further extension in Section 5 showed that incrementalism can be optimal for the agenda setter, even when there is some heterogeneity among the other legislators.

These results show that incrementalist budgeting practices can be explained in terms of the incentives of budget proposers to minimize the costs of building winning coalitions (and thereby maximize their payoffs). This provides an alternative perspective to Wildavsky's focus on cognitive limitations and the reduction of conflict. While this paper has shown that significant insights can be gained by analyzing public budgeting issues within a legislative bargaining framework, a number of further extensions have been left for future research. For instance, this paper follows the standard legislative bargaining approach and assumes that revenues are exogenous. However, it would be of interest to relax this assumption, and to examine the consequences of incrementalism for the aggregate size of the budget. ${ }^{22}$ Another issue that could be explored is whether a legislature that is choosing its institutional structure by majority vote would decide to permit institutions and rules that support incrementalism. ${ }^{23}$

It should also be noted that the model in Section 4 gives rise to a number of (potentially) testable predictions (illustrated numerically in Table 1). In particular, the higher is $q$ in relation to $n$, the less incentive exists for incremental budgeting; thus, supermajority requirements that reduce $A$ 's ability to exclude $j$ 's from the coalition will tend to decrease the payoff from incrementalism. In addition, the more patient are the legislators (i.e. the higher is $\delta$ ), the greater

\footnotetext{
${ }^{22}$ Tohamy (2000) examines Wagner's Law (of growth in government size) in the light of budgetary incrementalism.

${ }^{23}$ Recall that $A$ 's allocations are higher and each $j$ 's allocations are lower under incrementalism. This paper uses linear payoffs (an assumption that is innocuous here, as the results would generally be reinforced if payoffs were concave). If $A$ is chosen randomly before the first period, each legislator, voting in a prior organizational stage, would be indifferent between incremental and nonincremental budgeting (as $A$ 's gains are offset by the losses to the $j$ 's). However, this would not be true with concave payoffs, so this issue merits further exploration.
} 
the incentives for incremental budgeting. It would be an interesting exercise to confront these predictions with the available data. Other possible topics to explore include the impact of term limits on the ability to sustain coalitions over time, and the role of incrementalism in facilitating logrolling among legislators and among legislative committees.

\section{References}

Baron, D. (1998) "Comparative Dynamics of Parliamentary Governments", American Political Science Review, 92, 593-609.

Baron, D. and J. Ferejohn (1989) "Bargaining in Legislatures", American Political Science Review, 83, 1181-1206.

Berry, W. D. (1990) "The Confusing Case of Budgetary Incrementalism: Too Many Meanings for a Single Concept", Journal of Politics, 52, 167-196.

Crain, W. M. and N. V. Crain (1998) "Fiscal Consequences of Budget Baselines", Journal of Public Economics, 67, 421-436.

Davis, O. A., M. A. H. Dempster, and A. Wildavsky (1966) "A Theory of the Budgetary Process", American Political Science Review, 60, 529-547.

Dharmapala, D. (1999) "Comparing Tax Expenditures and Direct Subsidies: The Role of Legislative Committee Structure”, Journal of Public Economics, 72, 421-454.

Diermeier, D. and A. Merlo (2000) "Government Turnover in Parliamentary Democracies", Journal of Economic Theory, 94, 46-79.

Garrett, E. (1998) "Rethinking the Structures of Decisionmaking in the Federal Budget Process", Harvard Journal on Legislation, 35, 387-445.

Gist, J. R. (1982) “'Stability' and 'Competition' in Budgetary Theory”, American Political Science Review, 76, 859-872.

Kamlet, M. S. and D. C. Mowery (1987) "Influences on Executive and Congressional Budget Priorities, 1955-1981”, American Political Science Review, 81, 155-178.

Knight, B. (2002) "Bargaining in Legislatures: An Empirical Investigation”, unpublished manuscript.

Leblanc, W., J. M. Snyder, Jr., and M. Tripathi (1999) "Majority-Rule Bargaining and the Under Provision of Public Investment Goods", Journal of Public Economics, 75, 21-47.

Myerson, R. B. (1993) "Incentives to Cultivate Favored Minorities under Alternative Electoral Systems", American Political Science Review, 87, 856-869. 
Tohamy, S. (2000) "Wagner's Law: A Statistical Relationship or the Result of the Budgetary Process?", unpublished manuscript.

Tohamy, S., P. H. Aranson, and H. Dezhbaksh (1999) “An Economic Theory of the Budgetary Process", unpublished manuscript.

Tohamy, S., H. Dezhbaksh, and P. H. Aranson (2000) “A New Approach for Testing Budgetary Incrementalism", unpublished manuscript.

Wildavsky, A. (1984) The Politics of the Budgetary Process, 4th ed., Little, Brown and Co. [orig. 1964]

Wildavsky, A. (1988) The New Politics of the Budgetary Process, 1st ed., Scott, Foresman and Co.

Fig. 1: Timing of One Period of the Budgeting Game

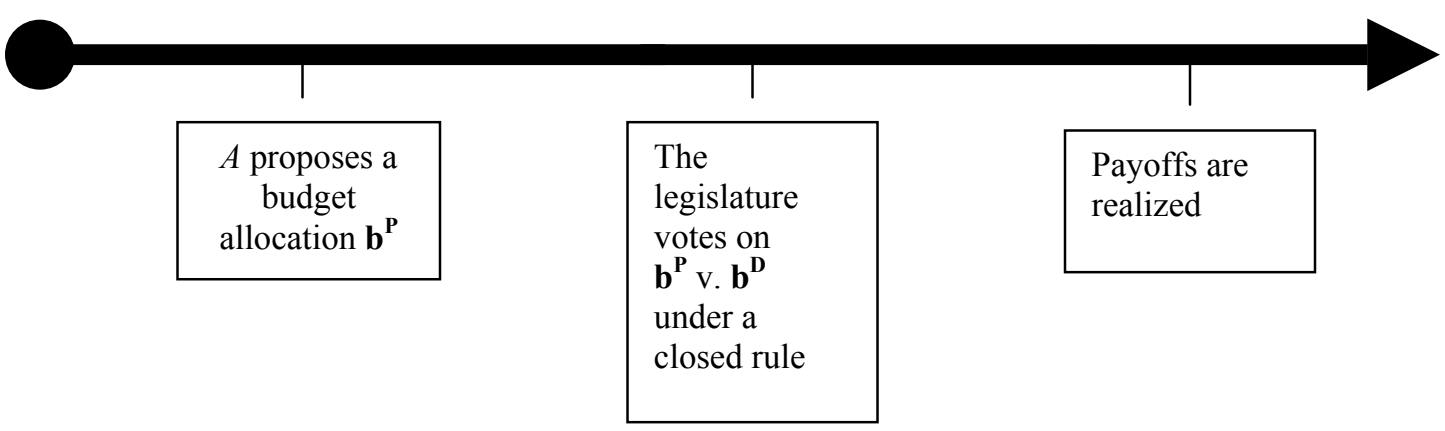

Table 1: The Magnitude of the Effect of Incremental Budgeting on $\gamma$

\begin{tabular}{|l|l|l|l|}
\hline $\boldsymbol{n}$ & $\mathbf{q}+\mathbf{1}$ & $\boldsymbol{\delta}$ & $\gamma$ (to 2 d.p.) \\
\hline 101 & 61 & 0.9 & 0.22 \\
\hline 101 & 61 & 0.99 & 0.02 \\
\hline 101 & 61 & 0.75 & 0.45 \\
\hline 101 & 81 & 0.9 & 0.36 \\
\hline 201 & 101 & 0.9 & 0.18 \\
\hline 435 & 218 & 0.9 & 0.18 \\
\hline
\end{tabular}

